\title{
Data processing in haematology
}

\author{
M. G. NELSON AND E. ELDER \\ From the Department of Haematology, Royal Victoria Hospital, Belfast
}

SYNOPSIS The output from a Coulter model $\mathrm{S}$ is captured by a specially designed silent interface $\stackrel{\overrightarrow{ }}{\circ}$ which visualizes the results, automatically prints the data on a continuous paper roll and transmits $\vec{\omega}$ the information to a card punch located in a data processing room. Further requested test data and $\stackrel{\Omega}{\Omega}$ patient identification data are subsequently added manually to the punch card. The completed deck용 of cards is used in an off-line batch mode on a dedicated laboratory computer to format report $N$ documents, produce ward listings, and quality control information. A punch card off-line method is $-\overrightarrow{ }$ also described for blood group and related data.

Automatic methods of data processing have been developed in the Clinical Pathology Laboratory at the Royal Victoria Hospital, Belfast.

At the present time a study is being carried out to test the feasibility of using both 'off-line' and 'on-line' loads on a dedicated laboratory computer. To this end the Division of Biochemistry has linked 18 channels to a multichannel continuous flow analytical equipment directly on-line to the computer, whilst the Divisions of Bacteriology and Haematology are processing batches of 80 -column cards during off-peak periods on the same computer.

In the Division of Haematology the methods of data processing have passed through a number of stages. In the first stage mark-sensed cards were used to produce 80-column punch cards via an interpreting machine but, for a variety of reasons, this system was rapidly abandoned. The second stage utilized the IBM 870 system which involved the manual generation of 80 -column punch cards. These were then passed through an IBM 836 control unit linked to a typewriter which produced worksheets and automatically typed report slips (Nelson, 1969). The present stage is an extrapolation of the IBM 870 system and involves an interface between the multichannel equipment (Coulter S) and the card punch, the manual addition of patient identification particulars (PID) and other test data, and the utilization of the laboratory IBM 1130 computer to format the report documents and produce a ward listing and quality control information.

The Division of Haematology provides a service for a teaching group of hospitals of 1480 beds in a 25-acre campus. In 1971 the total number of requests was 130965 or 358 requests per day on average and Received for publication 8 March 1972 the number of tests performed was 581574 which $\bar{\subseteq}$ represents 1593 tests per day on average. These requests were proportioned between the four sub- $\overrightarrow{0}$ sections of the haematology laboratory as follows: N routine 81 128; blood bank and immunohaematology 18 824; coagulation studies and anticoagulant control 26559; special haematological biochemistry 4454.

This short report is an account of the present $\stackrel{\varrho}{\circ}$ data processing system in the routine and blood $\stackrel{\varrho}{\rightarrow}$ bank subsections of the Department of Haematol-oํㅡㄹ ogy. It has been in daily use for more than a year and has proved capable of being married, without undue disruption, to the existing hospital request/ report system.

\section{Routine Haematology}

RECEPTION OF SPECIMENS

The laboratory porter carries out a 'milk round' of the various wards and departments twice a을. day and at 10.30 and 13.30 hours delivers these specimens to the various subsections of the labora- $\infty$ tory.

In the routine subsection the specimens are allo- N cated into two batches. The first batch contains 0 those for automatic multichannel analysis only and the second batch those specimens requiring manual tests. A unique laboratory accession number selected $\stackrel{\infty}{+}$ from a roll of sequential numbers, which is re-set 0 to 00001 on 1 January each year, is then put on each $\vec{P}$ specimen container and the accompanying request $\stackrel{\oplus}{\stackrel{\oplus}{ }}$ form. The batch for automated analysis is sequenced $\mathbb{Q}$ first, followed by the batch requiring manual tests. $\bar{\sigma}$ The accession number is used to correlate all 
activities relating to a specific blood specimen and to act as a specimen identification number.

\section{ANALYSIS SEQUENCE}

The majority of specimens are processed through a multichannel item of haematological equipment, namely, the Coulter model $\mathrm{S}$. The specimens are put through this equipment in order of laboratory accession number except for emergency requests, when the accession number on the instrument has to be altered manually to agree with that of the specimen. This equipment automatically produces seven haematological parameters with the accession number and the date. The output of the Coulter model S is interfaced directly to a card punch, consequently the test result data expressed in numerical form are automatically punched onto cards.

In order to calibrate the instrument, to check the results, and to provide results in response to urgent requests, it is necessary to have the data available at the bench in a readily accessible human sensible form, either on the conventional Coulter printout documents or printed on a continuous paper roll. A disadvantage of the Coulter printer is that the documents have to be fed into the printer at the same time as the specimen is being presented to the sampler probe. Because of this an automatic printer producing the data on a continuous paper roll has been developed. Additional advantages of the new system are that the test results may be reported in any order and as the data are stored may be reproduced more than once if required.

The present system has been designed to produce automatically on a punch card the laboratory accession number and the test data for the multichannel equipment. When this stage has been completed the patient identification particulars (PID) are manually added to each card by the punch card operator. The worksheet is automatically produced by passing the cards containing PID and Coulter S results through the IBM 836 punch linked to a typewriter using a hardwired program. Space exists on the worksheet for the addition of the results of manual tests. Each sample report occupies one line on the worksheet which is passed after production to the laboratory for the addition of the results of all the manual tests which have been requested.

The system just described requires the design of not only a suitable interface but also of an appropriate automatic printer. The interface module has been developed by Medical Scientific Computer Services, Belfast, and a strip printer obtained from Data Lab Limited.

THE INTERFACE

This incorporates the following features: (1) Visual display (in digital form) of the test results as they arise. (2) Test indication, ie, which test is being carried out on a specimen at any given point in time. (3) Automatic rounding of results to nearest significant figure on selected tests. (4) Driving circuitry for 545 card punch and associated timing signals for clearing and feeding in next card automatically. (5) An audio alarm in the event of a card jam (the card punch is in a data preparation room remote from the Coulter S). (6) An audio 'bleep' to signify to the Coulter $S$ technician that a card has been successfully punched, cleared, and the next card is in the waiting station. (7) Incorporation of a solid state memory system (capacity 32 digits) and circuitry to drive a Mullard type matrix printer (supplied by Data Lab Ltd). (8) Facility for not punching if required, eg, during 'wash-out' or calibration of the Coulter S. (9) The encoding of the Coulter $\mathrm{S}$ sequence number (set to the laboratory accession number) is also punched on the card. (10) Silent apart from 'bleep' as in (6) above. (11) Because all punching equipment is noisy the interface, which is silent in operation, has been located close to the analytical equipment but the IBM 545 punch is sited in a remote situation in the data processing room. This is $140 \mathrm{ft}$ away and is connected by a multicore cable to the interface. As a consequence appropriate signalling devices have been designed to indicate failure or fault in the punching sequence.

THE DATA PROCESSING SEQUENCE

As the specimens are being analysed the output from the Coulter $S$ is fed through the interface and the results are automatically punched onto cards which are processed as previously described.

The worksheet is moved from the data processing to the laboratory area where the data are edited and the results of manual tests are added. The completed worksheet is then returned to the data processing room for the punch card operator to add the manual test results to the appropriate card. At the same time any mistakes detected during editing are corrected and the appropriate cards removed from the deck and repunched. When a complete and accurate deck of cards is finally produced it is then passed in a batch to the IBM 1130 computer for further processing.

\section{OFFLINE BATCH PROCESSING ON THE} COMPUTER

The first batch of specimens is completed and the cards are punched by 12.30 hours. These are retained in the punch room until the second batch of cards is completed at $\mathbf{1 6 . 0 0}$ hours, then all the cards are taken to the computer building to be 
Data Processing Area
Laboratory

Area

RECEPTION

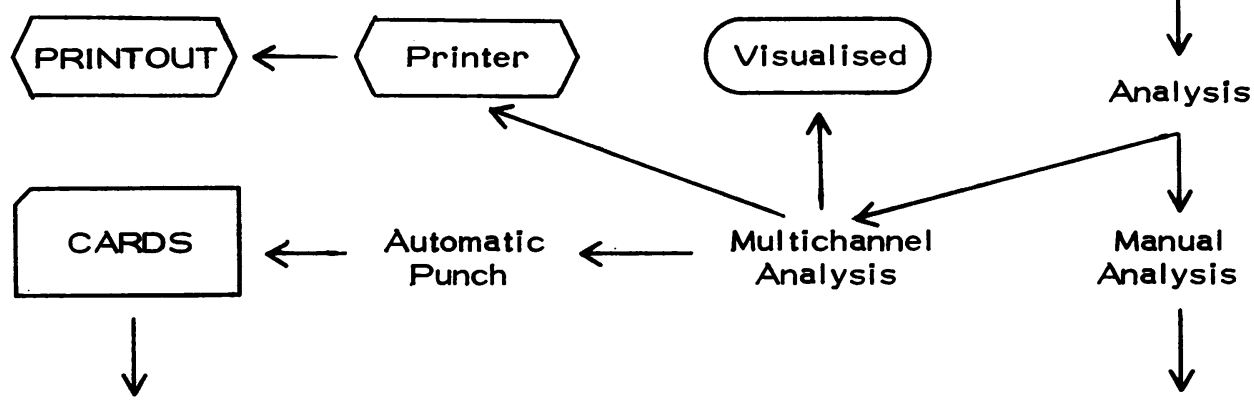

Specimen Batching

Accession Numbering

Addition of PID Worksheet Production

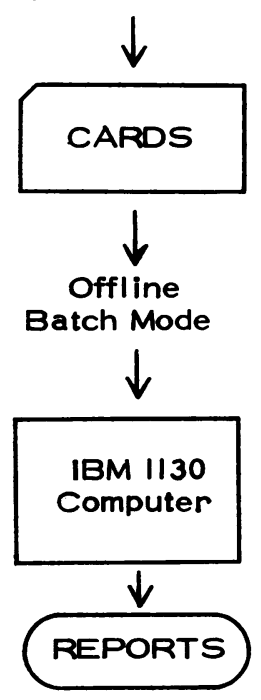

Worksheet Edited

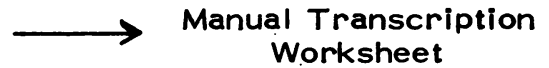

Manual Punch
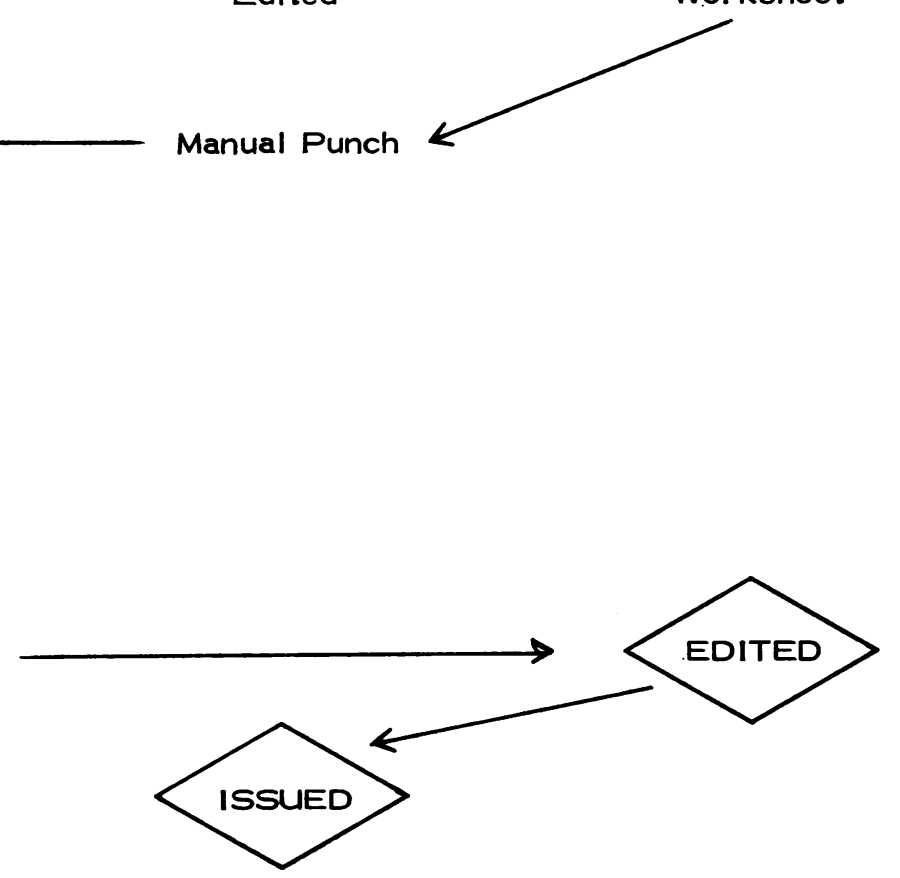

Fig. 1 Data processing flow diagram of routine haematology 
Data Processing

Area
Laboratory

Area

\section{RECEPTION}

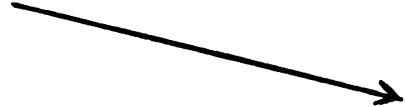

Specimen Separation

P.I.D. Accession Numbering Workshop

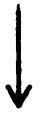

Analysis

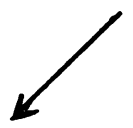

Antibody Screening

$A B O$ \& Rh. typing
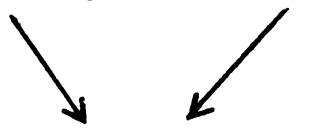

Manual Transcription

Worksheet

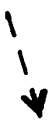

IBM 1130

Computer

$\downarrow$

Manual Punch

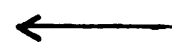

$\checkmark$

Preliminary Report

$A B O$ \& Rh. typing

\section{REPORTS}

$\downarrow$

enited
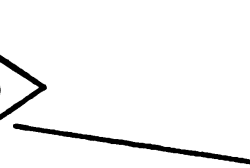

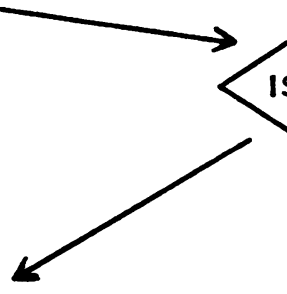

ISSUED

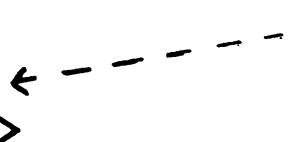

EDITED

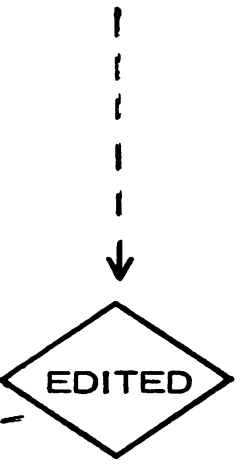

Fig. 2 Data processing flow diagram of blood

Pts. Ward Chart

Destroyed group information. 
processed by the laboratory IBM 1130 computer. A program has been written to produce a report for each patient on a $7 \times 5$ in. form in a vertical format with units and normal values automatically added. A program has also been written for the statistical analysis by the cusum method of the variation in haemoglobin and PCV results each day. The data from the cards are also used to produce, at the end of each day, a listing of all the results by wards and departments. This ward listing result sheet is used in the laboratory to answer telephone requests for results and a copy of the appropriate section is submitted to each of the ward units.

At present the number of cards in the batches varies from 150 to 300 per day. These can be processed in 30 to 45 minutes, the limiting factor being the speed of the line printer of the computer which produces an average of six reports per minute. The present arrangements permit the reports to be returned to the Department of Haematology by 17.00 hours when the various results can be checked against the day sheets, the reports sorted and then distributed to the wards.

\section{Hospital Blood Bank}

\section{RECEPTION OF SPECIMENS}

Specimens of blood and request/report forms reach the laboratory not only by the normal delivery after ward collection but also, depending on the degree of emergency, at irregular intervals during the day and night. The laboratory technician matches the request form with the appropriate blood specimen and a unique hospital blood bank laboratory accession number is given. This number is also entered onto the request form, serum tube and the laboratory worksheet. The PID is also entered onto the worksheet and onto the serum tube. This permits identification of the specimen and relates it to the patient.

\section{ANALYSIS OF SPECIMENS}

The ABO and Rhesus grouping is then carried out. The results are entered onto the worksheet and appropriately coloured self-adhesive labels affixed to the report part of the request/report document. This preliminary report is then sent to the ward.

All specimens of serum are screened for the presence of irregular antibodies and the results of these tests are also entered onto the worksheet.

DATA PROCESSING SEQUENCE

The following morning the worksheet is taken from the laboratory to the data processing area. Here the PID, blood bank laboratory accession number, and

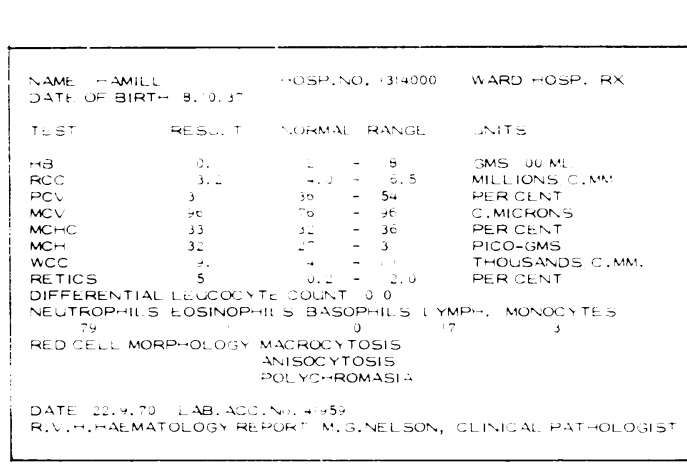

Fig. 3 Format of routine report.

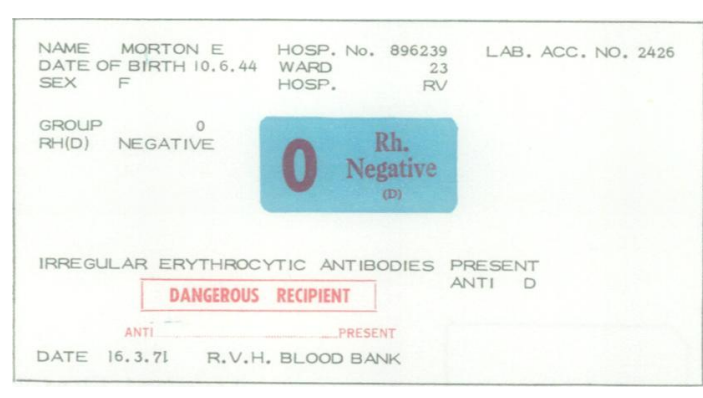

Fig. 4 Format of blood group report.

results of the tests are manually punched onto 80 흘 column cards.

When the batch of cards is complete, it is trans ferred to the computer area for further processing

BATCH PROCESSING OF CARDS ON THE COMPUTER

A program has been written to produce the bloodi group information and the results of the screening for irregular antibodies on a $7 \times 5$ in. report form.

EDITING OF REPORTS

When printed the reports are moved from the computer area back to the laboratory where the resultso are checked against the worksheet and at the samen time appropriately coloured blood group labels attached to the report forms. Where necessary gummed strips to indicate the presence of irregular antibodies are also attached. These reports are then submitted to the wards and units for incorporations in the patients' record documents. It is intended that the preliminary report already sent out should $\bar{\sigma}^{-}$ be destroyed and replaced by this automatically printed and edited report document.

Reference

Nelson, M. G. (1969). Punch card data processing in haematology. J. clin. Path., 22, 233-235. 